\title{
Severe abdominopelvic actinomycosis with colon perforation and hepatic involvement mimicking advanced sigmoid colon cancer with hepatic metastasis: a case study
}

Song Soo Yang and Yeong Cheol $\mathrm{Im}^{*}$

\begin{abstract}
Background: Actinomycosis is a rare chronic invasive disease caused by Actinomyces spp. Although abdominopelvic actinomycosis, which involves the colon and the pelvic organs extensively, has been frequently reported, abdominopelvic actinomycosis presenting with colon perforation and hepatic involvement concurrently has yet to be reported.

Case presentation: A 55-year-old woman presented at the emergency room with squeezing epigastric pain. Palpation of the abdomen revealed a hard mass with no acute peritoneal signs. Vital signs were normal range except for tachycardia. Initial laboratory testing revealed leukocytosis, anemia, elevated C-reactive protein (CRP), hypoalbuminemia; and normal AST/ALT and BUN/creatinine. CT scan of the abdomen-pelvis revealed a microperforations of the sigmoid colon, abscess in the left lower quadrant and hepatic lesion. Furthermore, there was a large infiltrating conglomerated mass invading the urinary bladder, left adnexa, sigmoid, left inguinal canal and left pelvic wall area. Ultrasound revealed an intra-uterine device (IUD). All these findings initially raised a suspicion of malignancy such as advanced cancer of the colon with liver metastasis. Despite the rarity of the disease, actinomycosis were not excluded because of the IUD found on ultrasound. Parenteral antibiotics and percutaneous drainage of abdomen abscess as well as fasting with total parental nutrition were prescribed for sigmoid perforation and abscess. After 10 days of conservative treatment, no remarkable change was detected in conglomerated mass invading pelvis. Furthermore, the finding of newly developed mechanical small bowel obstruction warranted surgery. Exploratory laparotomy was performed for the removal of perforated colon, obstructive small bowel and organs involved and postoperative histology confirmed a diagnosis of colonic actinomycosis. The patient made an uneventful recovery and was started on a 6-month course of penicillin.

Conclusions: Abdominopelvic actinomycosis presenting with colon perforation and hepatic involvement is extremely rare; however, it is clinically similar to advanced colon cancer with liver metastasis, therefore, complicating the preoperative diagnosis. A diagnosis of abdominopelvic actinomycosis should be considered in patients with a history of IUD and chronic abdominal pain, along with an abdominal mass or cutaneous abscess. If surgery is indicated, preoperative empirical antibiotic therapy for actinomycosis and frozen biopsy during surgery may be considered.
\end{abstract}

Keywords: Abdominopelvic actinomycosis, Colon perforation, Liver involvement

\footnotetext{
*Correspondence: driyc@hotmail.com

Department of Surgery, Ulsan University Hospital, University of Ulsan College

of Medicine, 877, Bangeojinsunhwando-ro, Dong-gu, Ulsan 44033, Republic

of Korea
}

(c) The Author(s). 2018 Open Access This article is distributed under the terms of the Creative Commons Attribution 4.0 International License (http://creativecommons.org/licenses/by/4.0/), which permits unrestricted use, distribution, and reproduction in any medium, provided you give appropriate credit to the original author(s) and the source, provide a link to the Creative Commons license, and indicate if changes were made. The Creative Commons Public Domain Dedication waiver (http://creativecommons.org/publicdomain/zero/1.0/) applies to the data made available in this article, unless otherwise stated. 


\section{Background}

Actinomycosis is a rare chronic invasive disease and Actinomyces israelii is the most prevalent species, anaerobic gram-positive bacteria that normally colonize oral, digestive and urogenital tracts in humans [1]. Breach of tissue integrity in mucosal lesions facilitates invasion of local structures and organs, leading to pathogenic co-infection with other organisms. All the tissues and organs may be infected, but four main clinical types of infection can be distinguished, depending on the primary site of infection: cervicofacial, thoracic, abdominopelvic, and disseminated disease [2]. Abdominopelvic actinomycosis is a rare disease encompassing abdominal infection, intrauterine devices (IUD)-related pelvic abscesses, infections of appendix, rectum and liver [3]. When it is associated with gastrointestinal organs, it is similar to chronic inflammatory bowel disease or malignancy, especially colon cancer [4]. Although abdominopelvic actinomycosis, which involves the colon and the surrounding pelvic organs extensively, has been frequently reported, abdominopelvic actinomycosis presenting with colon perforation and hepatic involvement concurrently has yet to be reported.

Here, we report a severe case of abdominopelvic actinomycosis with sigmoid colon perforation and hepatic lesion mimicking advanced colon cancer with liver metastasis.

\section{Case presentation}

A 55-year-old woman with no specific medico-surgical history presented at the emergency room with a 1-day history of squeezing epigastric abdominal pain. Patient also complained of a thick turbid yellowish discharge in the left inguinal area that was intermittently drained for some years.

Vital signs were normal range except for tachycardia (pulse rate, 110/min). Palpation of the abdomen revealed a wood-like hard mass in the left lower quadrant with minimal tenderness and no acute peritoneal signs warranting emergent surgery. A visible scar was noted in the left inguinal area without any discharge.

Initial laboratory testing revealed marked leukocytosis (white blood cells, 24,730 cells $/ \mathrm{mm}^{3}$ ), anemia (hemoglobin concentration of $6.9 \mathrm{~g} / \mathrm{dL}$ ), elevated C-reactive protein (CRP) $32.05 \mathrm{mg} / \mathrm{dL}$ (reference range, $0-0.5 \mathrm{mg} / \mathrm{dL}$ ), hypoalbuminemia (albumin, $2.5 \mathrm{~g} / \mathrm{dL}$ ); and normal AST/ALT and BUN/creatinine. CT scan of the abdomen-pelvis revealed a microperforation of the sigmoid colon, abscess in the left lower quadrant, a hepatic lesion and bilateral hydronephrosis. Furthermore, there was a large infiltrating heterogenous hyperattenuating conglomerated mass invading the urinary bladder, left adnexa, sigmoid, left inguinal canal and left pelvic wall area (Fig. 1). Ultrasound revealed an intra-uterine device (IUD) (Fig. 2). All these findings initially raised a suspicion of malignancy such as advanced cancer of the colon or ovary with liver metastasis. Despite the rarity of the disease, infectious diseases such as actinomycosis were not excluded because of the IUD found on ultrasound. Colonoscopy or percutaneous needle biopsy was not performed for accurate diagnosis due to suspected colon perforation and the small bowel enclosed mass.

Since the patient showed minimal peritoneal irritation and stable vital signs, and extensive organ resection was expected due to invasion of bladder and ureters, treatment was initially conservative rather than primary debulking surgery. The antibiotic regimen was always

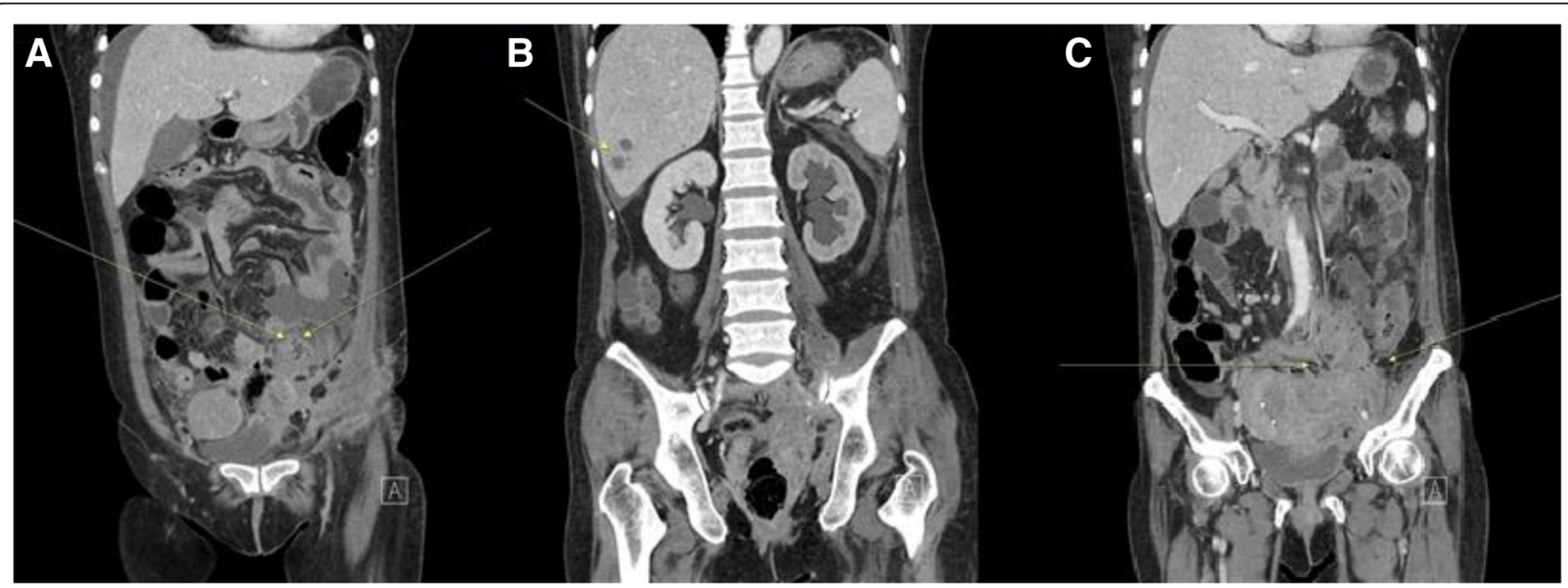

Fig. 1 CT finding at the emergency room. a CT scan of the abdomen-pelvis revealed a microperforation (arrow) of the sigmoid colon and abscess in the left lower quadrant. b CT scan showed a hepatic lesion (arrow) and bilateral hydronephrosis. c There was a large infiltrating heterogenous hyperattenuating conglomerated mass invading the urinary bladder, left adnexa, sigmoid, left inguinal canal and left pelvic wall area (arrow) 


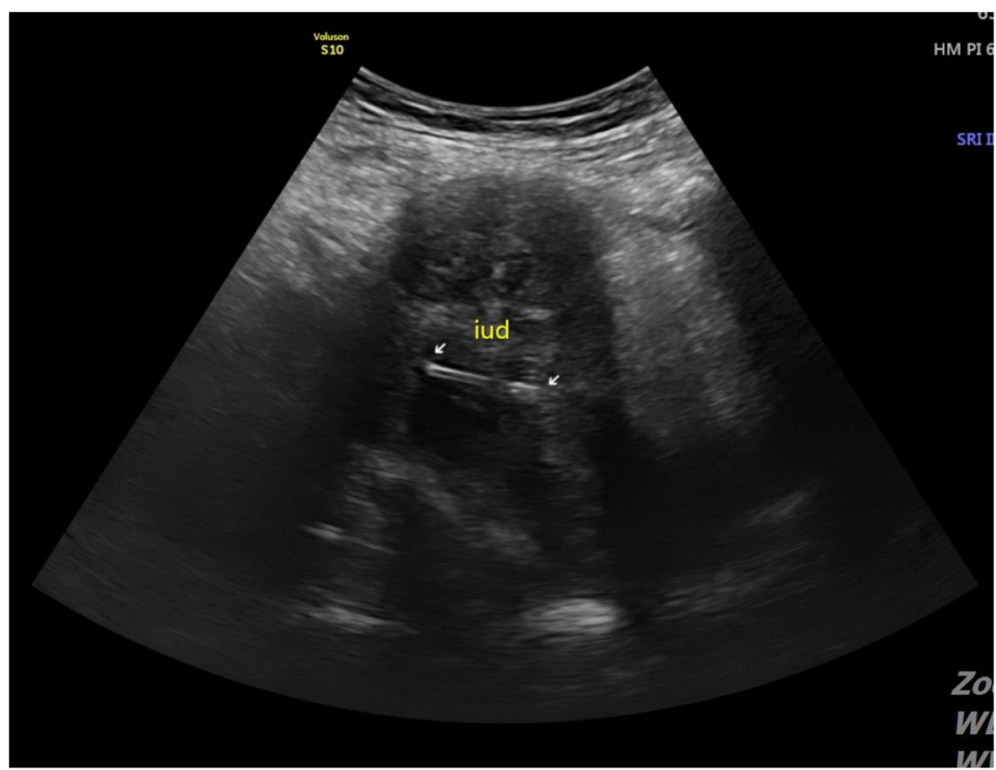

Fig. 2 Ultrasound revealed an intra-uterine device (IUD)

determined based on the infectious disease diagnosis after hospitalization. Parenteral antibiotics (ceftriaxone +metronidazole+azithromycin) and fasting with total parental nutrition were prescribed for sigmoid perforation. Because there was a huge left abdominal abscess $(11 \mathrm{X} 8 \mathrm{X} 3 \mathrm{~cm})$ that could spread to other spaces and cause generalized peritonitis, the imaging-guided percutaneous abscess drainage was performed.

After 10 days of conservative treatment, a repeat CT scan of the abdomen-pelvis showed improvement in abdominal abscess and liver lesion. However, no remarkable change was detected in conglomerated mass invading pelvis. Furthermore, the finding of newly developed mechanical small bowel obstruction warranted surgery.

Exploratory laparotomy was performed for the removal of perforated colon, obstructive small bowel and organs involved. Abscess of the sigmoid colon involved the uterus, adnexa, loop of small bowel and distal colon with severe adhesion between the mass and pelvic organs including the uterus, small and large bowels, and bladder. The abscess compressed the left ureter and caused ureteral dilatation. En-bloc excision of the mass was performed using Hartmann's procedure, bilateral salpingo-oophorectomy, small bowel resection and appendectomy. The gynecologist decided not to resect uterus because of severe fibrotic adhesion in the pelvis and transvaginal IUD removal failed repeatedly due to severe adhesion.

Since the frozen section excluded malignancy, a double J catheter was inserted into both the ureters without resection. Although Actinomyces spp. failed to grow in preoperative cultures, postoperative permanent histology confirmed a definitive diagnosis of colonic actinomycosis, which showed the granular colonies of bacteria, commonly termed sulfur granule, with aggregates of filamentous bacteria and neutrophils (Fig. 3) and abscess with invasion into the uterus and ovaries.

After surgery, the parenteral antibiotic regimen was changed to tigecycline, amikacin, metronidazole and Penicillin G. Three days after surgery, bowel movement was restored and vital signs were stabilized, which decreased the abdominal pain. The patient made an uneventful recovery and was started on a 6-month course

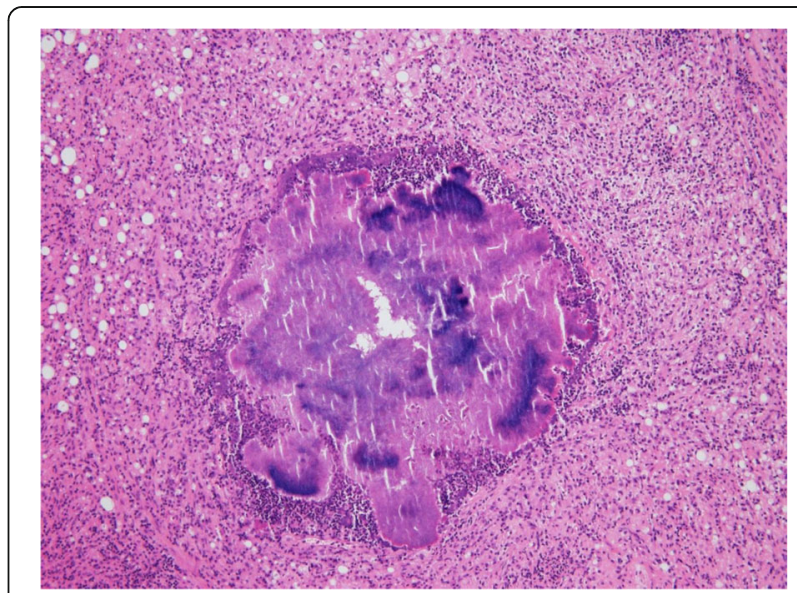

Fig. 3 This histologic section showed the granular colonies of bacteria, commonly termed sulfur granule, with aggregates of filamentous bacteria and neutrophils. H\&E × 100 
of penicillin. At 1-year follow-up, the patient was well and free from disease.

\section{Discussion and conclusions}

Abdominopelvic actinomycosis is one of the main clinical types according to the site of infection. It is a rare disease, but it leads to tissue granulation, dense fibrosis and abscess, resulting in a hard pelvic mass compressing the urinary bladder, ureter and rectum [5]. Previous studies of actinomycosis showed bowel strictures or hydronephrosis [6, 7]. However, whatever the origin of infection, colon perforation is a rare event. There is a case that a single perforation of the transverse colon at the hepatic flexure caused by obstructing sigmoid colon mass was managed by emergency surgery and subsequent histologic examination revealed actinomycosis [8]. In our case, the precise factors underlying spontaneous colon perforation are unclear. However, it is assumed that the high pressure of the proximal colon due to the colorectal stricture as well as persistent inflammation of the sigmoid colon wall due to actinomycosis triggered colon perforation.

Hepatic actinomycosis (HA) is also a very rare form of abdominal actinomycosis and often, it is a secondary infection following abdominal infection. HA constitutes $15 \%$ of abdominal actinomycosis, and $5 \%$ of all actinomycosis [9-11]. The clinical features, diagnosis and treatment of colonic and hepatic actinomycosis in previous cases were shown in Table 1.

In the present unusual case, the patient presented with signs and symptoms mimicking advanced colon cancer with liver metastasis. Few studies have demonstrated other clinical features such as mimicking colon cancer, large mass with perforation, hydronephrosis and involvement of adjacent tissues. However, there are no reports showing all the features including colon perforation, rectal stricture, hydronephrosis, ascites and hepatic involvement.

Abdominopelvic actinomycosis does not produce the characteristic disease signs or symptoms and usually manifests as a slowly growing mass, which may be associated with altered bowel habits, nausea, vomiting and cramping pain [12]. Patients with hepatic involvement present mostly with chronic or subacute and non-specific symptoms including anorexia, weight loss, fever and night sweats $[11,13]$. The nonspecific findings complicate the differential diagnosis of abdominopelvic actinomycosis from other chronic diseases such as chronic granulomatous infection, inflammatory bowel disease, and pelvic inflammatory diseases. Furthermore, it often presents as a mass either clinically or radiologically, which is not easily distinguishable from malignancies $[12,14,15]$.

Several reports show abdominopelvic actinomycosis mimicking malignancies $[16,17]$. Due to the misdiagnosis, several previous cases were treated with neoadjuvant chemotherapy [17-19]. Hepatic actinomycosis is also difficult to distinguish from primary hepatocellular carcinoma and metastatic liver cancer [20].

In addition, because the preoperative diagnosis is rarely considered and is established only in less than $10 \%$ of cases, the diagnosis is based on clinical manifestations and imaging findings $[12,21]$. Since the cultures of Actinomyces species show very low yield, histopathological examination is the most utilized diagnostic method worldwide, which is generally conducted after surgical intervention due to an initial diagnostic error $[4,12,22]$. Sulfur granules were observed in the purulent material in 50\% of cases. Although these might not be pathognomonic of actinomycosis, the presence of sulfur granules is highly suggestive of the diagnosis $[23,24]$.

In our case, colon perforation with liver lesion resembled advanced colon cancer with liver metastasis. Radiologically, actinomycosis was considered, but colon cancer with liver metastasis was not excluded.

Treatment of abdominopelvic actinomycosis depends on the extent of the disease and the patient's condition. Long-term treatment with penicillin is the standard medical therapy for uncomplicated cases [25]. Indeed, Actinomyces spp. are usually extremely susceptible to beta-lactams, and especially Penicillin G or amoxicillin. Clindamycin, tetracycline, and erythromycin are alternatives in cases of allergy to penicillin [12]. Piperacillin-tazobactam or a carbapenem (imipenem or meropenem) may be an appropriate alternative [26]. The need for surgery must be assessed on an individual basis and surgery may be a valid option for patients who do not respond to medical treatment [26].

Treatment of HA mainly involves surgical or puncture drainage, hepatic resection, and postoperative treatment with anti-infectives $[9,20]$.

This particular case is interesting in several aspects.

First, although preoperative antibiotic therapy was administered only for 2 weeks, a significant reduction in inflammation due to actinomycosis was detected during surgery. The bladder and ureter, which were expected to be sacrificed, were preserved. Ureteral dilatation and hydronephrosis were resolved following insertion of a temporary double-J stent, and antibiotic therapy as reported previously [27-29].

Second, the exclusion of malignant tumors in frozen biopsy during surgery also facilitated the demarcation of the extent of surgery. In most of the previous cases, abdominopelvic mass was considered as a malignant tumor before surgery, and the diagnosis of actinomcycosis was made after surgery $[2,4,8,18,19,28-31]$. However, in our case, by excluding the malignant tumor through the frozen biopsy during surgery, we could avoid unnecessary extensive surgery. 


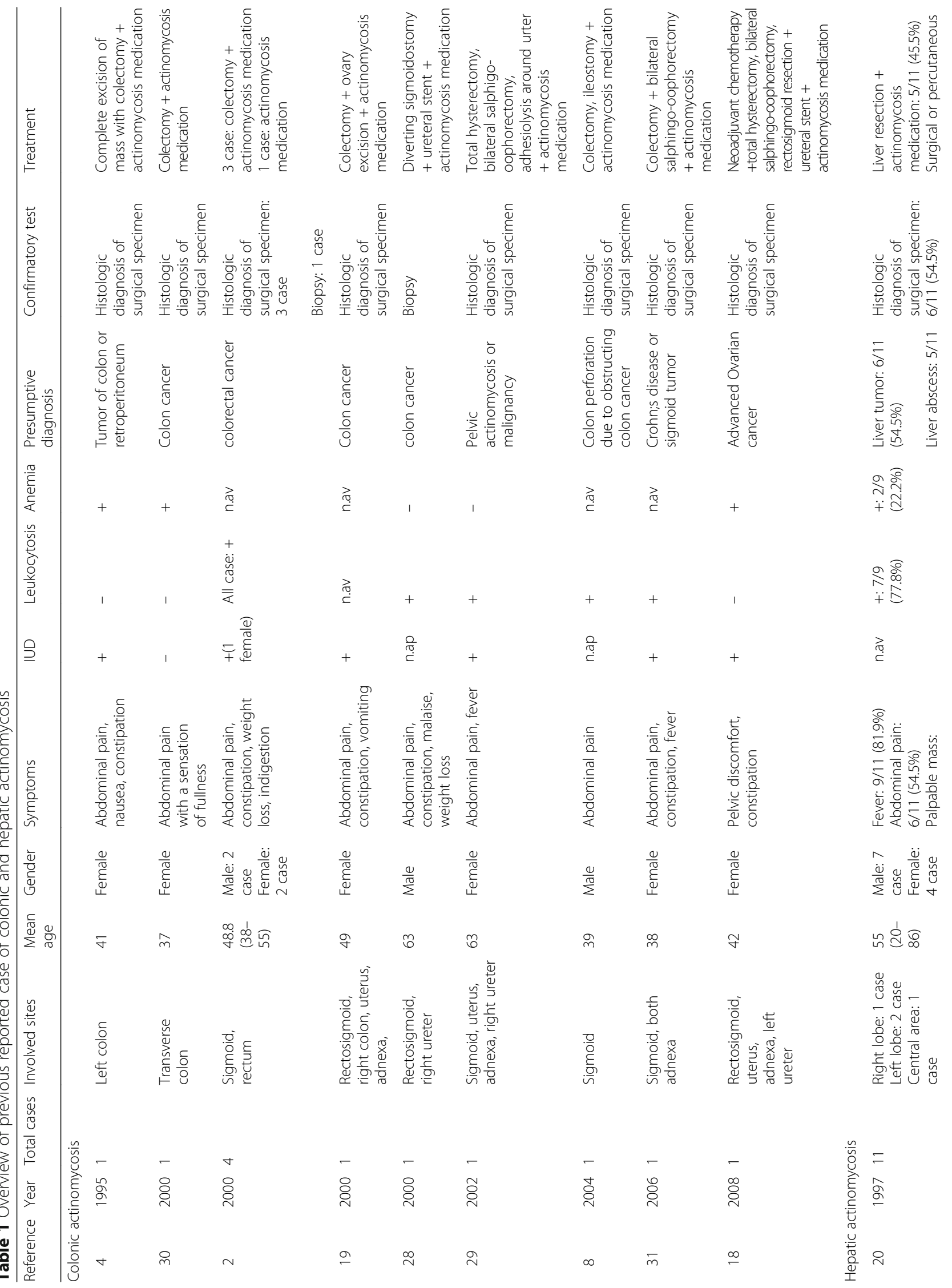




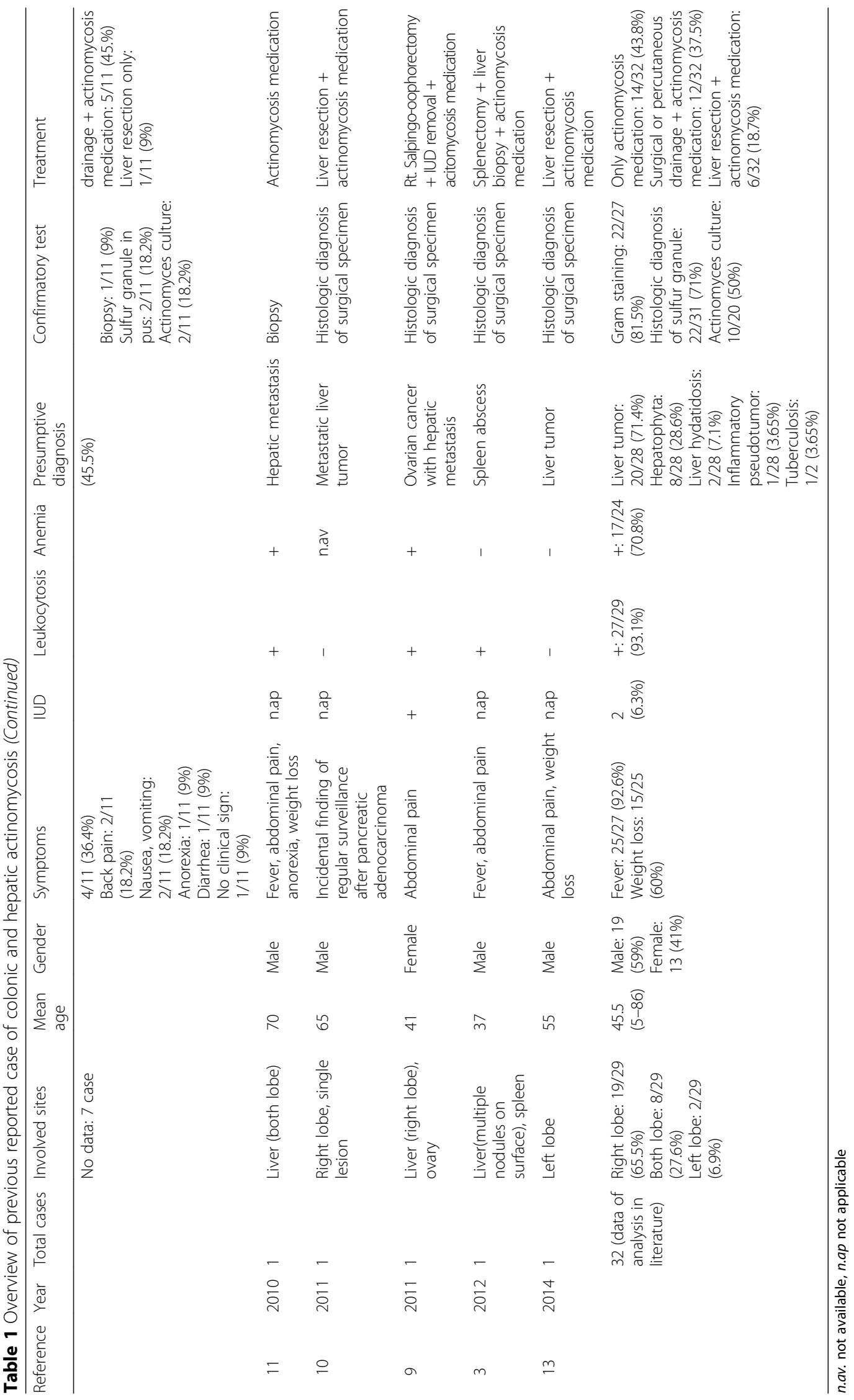


Therefore, we recommend the use of preoperative empirical antibiotics and exclusion of malignant tumors during surgery via frozen biopsy. Such a strategy reduces the extent of surgery and postoperative complications in patients, with actinomycosis indistinguishable from malignant tumor before surgery.

In conclusion, abdominopelvic actinomycosis presenting with colon perforation and hepatic involvement is extremely rare; however, it is clinically similar to advanced colon cancer with liver metastasis, therefore, complicating the preoperative diagnosis. A diagnosis of abdominopelvic actinomycosis should be considered in patients with a history of IUD and chronic abdominal pain, along with an abdominal mass or cutaneous abscess. If surgery is indicated, preoperative empirical antibiotic therapy for actinomycosis and frozen biopsy during surgery may be considered.

\section{Author's ex-post considerations}

- A diagnosis of abdominopelvic actinomycosis should be considered in patients with a history of IUD, even though concurrent hepatic mass was detected.

- If the patient's condition allows, the use of preoperative empirical antibiotics should be considered for at least 2 weeks to decrease the extent of surgery and postoperative complications.

- If surgery is indicated, exclusion of malignant tumors via intraoperative frozen biopsy facilitated the determination of the extent of surgery.

\section{Abbreviations}

ALT: Alanine transaminase; AST: Aspartate transaminase; BUN: Blood urea nitrogen; CRP: C-reactive protein; CT: Computer tomography; HA: Hepatic actinomycosis; IUD: Intra-uterine device

\section{Availability of data and materials}

The datasets used and/or analyzed during the current study are available from the corresponding author on reasonable request.

\section{Authors' contributions}

SSY and YCI drafted the manuscript. Both authors have read and approved the manuscript, and ensure that this is the case.

\section{Ethics approval and consent to participate}

Not applicable.

\section{Consent for publication}

Written informed consent was obtained from the patient for the publication of this case report.

\section{Competing interests}

The authors declare that they have no competing interests.

\section{Publisher's Note}

Springer Nature remains neutral with regard to jurisdictional claims in published maps and institutional affiliations.
Received: 11 May 2018 Accepted: 27 July 2018

Published online: 02 August 2018

\section{References}

1. Berardi R. Abdominal actinomycosis. Surg Gynecol Obstet. 1979;149:257-66.

2. Son SH, Kim BS, Huh KC, Park SK. Abdominal actinomycosis initially diagnosed as a colorectal cancer or periappendiceal abscess. Korean $J$ Gastrointest Endosc. 2000;21(3):717-22.

3. Wang H-K, Sheng W-H, Hung C-C, Chen Y-C, Liew P-L, Hsiao C-H, Chang SC. Hepatosplenic actinomycosis in an immunocompetent patient. J Formos Med Assoc. 2012;111(4):228-31.

4. Kaya E, Yilmazlar T, Emiroğlu Z, Zorluoğlu A, Bayer A. Colonic actinomycosis: report of a case and review of the literature. Surg Today. 1995;25(10):923-6.

5. Fiorino AS. Intrauterine contraceptive device-associated actinomycotic abscess and Actinomyces detection on cervical smear. Obstet Gynecol. 1996;87(1):142-9.

6. Bae JJ, Kim JH, Park YK, Lee DJ, Koh MW, Lee TH, Lee SH. A clinical analysis of pelvic actinomycosis. Korean J Obstet Gynecol. 2007;50(8):1132-40.

7. Hyung WJ, Kim MW, Kim MK, Chang DY, Jeon MK, Lee ES. 4 cases of pelvic actinomycosis associated with intrauterine contraceptive device. Korean J Obstet Gynecol. 2005;48(2):509-18.

8. Norwood MG, Bown MJ, Furness PN, Berry DP. Actinomycosis of the sigmoid colon: an unusual cause of large bowel perforation. ANZ I Surg. 2004;74(9):816-8.

9. Kim YS, Lee BY, Jung MH. Metastatic hepatic actinomycosis masquerading as distant metastases of ovarian cancer. J Obstet Gynaecol Res. 2012;38(3): 601-4.

10. Wayne MG, Narang R, Chauhdry A, Steele J. Hepatic actinomycosis mimicking an isolated tumor recurrence. World J Surg Oncol. 2011;9(1):70.

11. Kanellopoulou T, Alexopoulou A, Tiniakos D, Koskinas J, Archimandritis AJ. Primary hepatic actinomycosis mimicking metastatic liver tumor. J Clin Gastroenterol. 2010;44(6):458-9.

12. Garcia-Garcia A, Ramirez-Duran N, Sandoval-Trujillo H, Romero-Figueroa MDS. Pelvic Actinomycosis. Can J Infect Dis Med Microbiol. 2017;2017: 9428650.

13. Yang $X X$, Lin JM, Xu KJ, Wang SQ, Luo $T$, Geng $X X$, Huang $R G$, Jiang $N$. Hepatic actinomycosis: report of one case and analysis of 32 previously reported cases. World J Gastroenterol. 2014;20(43):16372-6.

14. Lim DR, Hur H, Min BS, Baik SH, Kim NK. Intrauterine contraceptive devicerelated actinomycosis infection presenting as ovarian cancer with carcinomatosis. Surg Infect. 2014;15(6):826-8.

15. Weese WC, Smith IM. A study of 57 cases of actinomycosis over a 36-year period: a diagnostic 'failure' with good prognosis after treatment. Arch Intern Med. 1975;135(12):1562-8.

16. Hoffman M, Roberts W, Solomon P, Gunasekarin S, Cavanagh D. Advanced actinomycotic pelvic inflammatory disease simulating gynecologic malignancy. A report of two cases. J Reprod Med. 1991;36(7):543-5.

17. Koshiyama M, Yoshida M, Fujii H, Nanno H, Hayashi M, Tauchi K, Kaji Y. Ovarian actinomycosis complicated by diabetes mellitus simulating an advanced ovarian carcinoma. Eur J Obstet Gynecol Reprod Biol. 1999;87(1): 95-9.

18. Lee YK, Bae JM, Park YJ, Park SY, Jung SY. Pelvic actinomycosis with hydronephrosis and colon stricture simulating an advanced ovarian cancer. J Gynecol Oncol. 2008;19(2):154-6.

19. Yeguez JF, Martinez SA, Sands LR, Hellinger MD. Pelvic actinomycosis presenting as malignant large bowel obstruction: a case report and a review of the literature. Am Surg. 2000;66(1):85.

20. Sugano S, Matuda T, Suzuki T, Makino H, linuma M, Ishii K, Ohe K, Mogami K. Hepatic actinomycosis: case report and review of the literature in Japan. J Gastroenterol. 1997;32(5):672-6.

21. Chan P, Chong S, Ng B, Chan S. Splenic actinomycosis. J R Coll Surg Edinb. 1999:44(5):344

22. Smith TR. Actinomycosis of the distal colon and rectum. Gastrointest Radiol. 1992;17(1):274-6.

23. Cintron JR, Del Pino A, Duarte B, Wood D. Abdominal actinomycosis. Dis Colon Rectum. 1996;39(1):105-8.

24. Chitturi S, Hui J, Salisbury E, Mitchell D, George J. Abdominal pain in an intrauterine contraceptive device user. Postgrad Med J. 2001;77(911):602.

25. Valour F, Senechal A, Dupieux C, Karsenty J, Lustig S, Breton P, Gleizal A, Boussel L, Laurent F, Braun E, et al. Actinomycosis: etiology, clinical features, diagnosis, treatment, and management. Infect Drug Resist. 2014;7:183-97. 
26. Wong V, Turmezei T, Weston V. Actinomycosis. BMJ. 2011;343:d6099.

27. Fulton I, Paterson W, Crucioli V. Pelvic actinomycosis causing ureteric obstruction. BJOG Int J Obstet Gynaecol. 1981;88(10):1044-50.

28. Haj M, Nasser G, Loberant N, Cohen I, Nesser E, Eitan A. Pelvic actinomycosis presenting as ureteric and rectal stricture. Dig Surg. 2000;17(4):414-7.

29. Nasu K, Matsumoto H, Yoshimatsu J, Miyakawa I. Ureteral and sigmoid obstruction caused by pelvic actinomycosis in an intrauterine contraceptive device user. Gynecol Obstet Investig. 2002;54(4):228-31.

30. Kim H, Lee WK, Kim SH, Pak SM, Kim YN, Choi SJ, Kim EH, Choi YW, Lee YU A case of abdominal actinomycosis. Korean J Gastroenterol Endo. 2000; 20(4):307-11.

31. Valko P, Busolini E, Donati N, Chimchila Chevili S, Rusca T, Bernasconi E. Severe large bowel obstruction secondary to infection with Actinomyces israelii. Scand J Infect Dis. 2006;38(3):231-4.

Ready to submit your research? Choose BMC and benefit from:

- fast, convenient online submission

- thorough peer review by experienced researchers in your field

- rapid publication on acceptance

- support for research data, including large and complex data types

- gold Open Access which fosters wider collaboration and increased citations

- maximum visibility for your research: over $100 \mathrm{M}$ website views per year

At BMC, research is always in progress.

Learn more biomedcentral.com/submissions 